\title{
Transcriptome Analysis of Gene in Mouse M-1 Cells Revealing the Functional Mechanism of Astragali Radix Extract
}

\author{
Bing Wang*, Qian Feng*, Chao Zhang, Yuming Chen, Mengmei Li, Yu Chen, Lin Wang, Jie Teng, \\ Zhongzhen Cai, Yanpeng Zhao, Xuemei Bai"
}

ENNOVA Institute of Life Science and Technology, ENN Group, Langfang, China

Email: "baixuemei@enn.cn

How to cite this paper: Wang, B., Feng, Q., Zhang, C., Chen, Y.M., Li, M.M., Chen, Y., Wang, L., Teng, J., Cai, Z.Z., Zhao, Y.P. and Bai, X.M. (2021) Transcriptome Analysis of Gene in Mouse M-1 Cells Revealing the Functional Mechanism of Astragali Radix Extract. Pharmacology \& Pharmacy, 12, 293-310.

https://doi.org/10.4236/pp.2021.1212025

Received: November 26, 2021

Accepted: December 21, 2021

Published: December 24, 2021

Copyright $\odot 2021$ by author(s) and Scientific Research Publishing Inc. This work is licensed under the Creative Commons Attribution International License (CC BY 4.0).

http://creativecommons.org/licenses/by/4.0/

\begin{abstract}
Astragali Radix (AR), the dried root of legumes, belongs to the Qi-invigorating herbs in traditional Chinese medicine and plays an important role in the treatment of many diseases. In order to understand the mechanism of action of AR extract. We used AR extract to treat M-1, mouse kidney cells, and used transcriptome sequencing technology to detect the genomic transcription level of the cells under the action of AR at different concentrations and times. The results showed that after a low concentration of AR treatments on the cells, the expression of genes related to cell growth and cellular immune response changed significantly, among which multiple genes are related to mitochondrial function, while high concentrations of AR affected the expression of histones and disease-related genes. It showed that the low concentration of AR extract can achieve the effect of invigorating Qi by regulating the function of mitochondria. In addition, several important genes and pathways were identified as potential targets of AR activation. The research not only clarified the main molecular biological mechanism of AR invigorating Qi, but also provided experimental basis and cellular physiology reference for the further clinical application of AR.
\end{abstract}

Keywords

Astragali Radix, RNA-Seq, Qi, Mitochondria

\section{Introduction}

Astragali Radix (AR), a perennial leguminous herb whose root is used as medi*The authors Bing Wang and Qian Feng contributed equally to the work.

\#Corresponding author. 
cine, is one of the most common and popular Chinese herbal medicines. In more than a thousand years of the development of the traditional Chinese medicine, people have discovered many drugs for invigorating $\mathrm{Q} i$, and AR is a representative one for Qi invigoration. Based on the important role of AR, a variety of AR medicinal products have been developed, including Astragalus tablets, Astragalus Polysaccharide Granules, Astragalus Glucoside Granules, etc., which have been used in the treatment of many diseases, including COVID-19, a novel coronavirus pneumonia pandemic, occurred in early 2020 . The combined use of Astragalus and other Chinese herbal medicines played an important role in the prevention and treatment of COVID-19 infections [1], saving many lives and reducing economic losses.

A variety of natural plant products can be extracted from AR, mainly containing Astragaloside, Astragalus flavonoids, Astragalus polysaccharides (APS) and amino acids. Astragaloside IV can significantly reduce myocardial damage caused by ischemia and hypoxia, improve energy metabolism, improve endothelial dysfunction, promote angiogenesis, improve immune function, and regulate blood sugar and blood lipid levels [2]. Flavonoids are mainly ingested as glycosides, which have exhibited many potential beneficial activities in animal research and human trials [3]. APS is a type of water-soluble heteropolysaccharide with biological activity, which has the characteristics of low toxic and side effects, no residue and intolerance. The biological functions of APS include regulating immune function, anti-aging, anti-tumor [4], lowering blood sugar, lowering blood fat, anti-fibrosis, antibacterial, radiation protection and antiviral effects [5]. AR extract was the isolated effective ingredient from AR dry root, and its biological effects were as effective as dry AR root.

In the literature, the existing high-throughput sequencing studies related to $A R$ are either the analysis of the therapeutic effects of the main components of AR on disease models [6] [7], or the analysis of the effects of AR extracts on plants development [8]. Except for the transcriptome analysis of compound medicines containing AR [9], there is no transcriptome analysis research on the effect of AR extract to illustrate the healing effect of AR. For this study, we applied the extract of AR, a traditional Chinese medicine for invigorating Qi, to mouse M-1 cells, and used modern transcriptomics methods to screen differently expression genes during cell culture to study the effects of traditional Chinese medicine on cells. It is a bridge between traditional medicine and modern medical research, which helps us to understand the functional mechanism of the Qi-invigorating herb.

\section{Method}

\subsection{Extraction of Astragali Radix}

Astragalus root was purchased from a Chinese medicine store (Tongrentang chain-store), and sliced (50 g) to pieces and powdered. The powder was soaked in $500 \mathrm{ml}$ distilled water for $1 \mathrm{~h}$ and then boiled at $100^{\circ} \mathrm{C}$ for $30 \mathrm{~min}$. The mixed 
liquid was filtered to get the supernatant, and the insoluble matter was again mixed with $400 \mathrm{ml}$ distilled water and boiled at $100^{\circ} \mathrm{C}$ for $20 \mathrm{~min}$. The supernatant was obtained by the same way and mixed with the former supernatant. Extracting solution was concentrated by rotary evaporation apparatus, which concentrated AR extract corresponds to the dry weight of AR $0.7 \mathrm{~g} / \mathrm{ml}$.

\subsection{Cells Culture and Treatment}

Mouse cell line M1 (ATCC, CRL-2038, USA) was grown in Dulbecco's modified Eagle's medium (DMEM, Thermo, USA) supplemented with $10 \%$ fetal bovine serum (FBS, Thermo, USA) and 1\% penicillin-streptomycin. Cells were cultured at $37^{\circ} \mathrm{C}$ in a $5 \% \mathrm{CO}_{2}$ incubator. During the experimental period, cells were divided into three groups: low dose, high dose and control group. The cell medium was added with a AR extract to a final concentration of $175 \mu \mathrm{g} / \mathrm{ml}$ (low dose) and $875 \mu \mathrm{g} / \mathrm{ml}$ (high dose). Low-dose cells were incubated for $4 \mathrm{~h}$ and $24 \mathrm{~h}$, and the high-concentration cells were incubated for $4 \mathrm{~h}$, followed by removing of AR supernatant. Cells with no AR treatment were used as blank control, and phosphate buffered saline (PBS, Thermo, USA) was used instead of extract. The cells were trypsinized, washed with PBS, and the cell pellet was collected and washed with PBS again. The experiment was conducted in three biological replicates.

\subsection{RNA Extraction and Construction of cDNA Library}

Isolate total RNA from M-1 cells treated by AR extraction according to the manufacturer's instructions of TaKaRa MiniBEST Universal RNA Extraction Kit (9767, Japan). The quantity and purity of RNA was assessed by Agarose gel electrophoresis. RNA was then sent to Sangon Biotech (Shanghai) Co., Ltd for transcriptome analysis. Sequences were initial analyzed using Illumina HiSeqTM system, Shanghai Sangon Biotech, Shanghai, China.

\subsection{Library Quality Identification and Sequence Comparison Analysis}

The quality of raw data, sequences obtained from the Illumina HiSeqTM system, were further assessed by FastQC software. Nucleic acid sequences containing low quality base, unknown base, etc. were excluded. The remaining sequences after quality control were compared with the reference genome of Mus musculus mm10. Expression abundance of genes was calculated in terms of transcripts per million (TPM). Data were obtained form 3 independent biological replicates.

\subsection{Screening of Differentially Expressed Genes}

DEGs were analyzed by DEGseq software. Genes with $\mathrm{p}$ value $<0.05$ and $\log _{2}$ (fold change) $>2$ were considered significantly differently expressed genes (DGEs). Significant DEGs in all groups were calculated and shown in Volcano and Veen map. The overlap of Veen map represented the common DEGs (co-DEGs) be- 
tween groups and indicated the connection of DEGs from each group. Numbers in Veen map represented the unique or universal DEGs between groups.

\subsection{Analysis of GO and KEGG}

The molecular function, subordinate cellular component and involved biological process of DEGs were analyzed using the OmicShare tools by Gene Ontology (GO). Kyoto Encyclopedia of Genes and Genomes (KEGG) database were used for functional annotation of the DEGs.

\section{Result}

\subsection{Transcriptome Analysis of the Effect of Low-Dose AR on M-1 Cells}

In order to reveal the molecular mechanism of the effects of AR on cells after different periods of exposure time, the low-dose AR extract $(175 \mu \mathrm{g} / \mathrm{ml})$ was added to the cell culture medium, and the cell pellets were collected at two time points of $4 \mathrm{~h}$ and $24 \mathrm{~h}$. Use high-throughput sequencing to remove predicted genes (gene names start with "GM" and then coded with numbers), and compare gene expression changes between the control group and treatment group at the same time point. The results showed that 439 DEGs (including 233 up-regulated genes and 206 down-regulated genes) and 475 (including 268 up-regulated genes and 207 down-regulated genes) were screened out between $4 \mathrm{~h}$ and $24 \mathrm{~h}$ after AR treatment ( $\mid$ fold change $\mid>2$ and $\mathrm{p}<0.05$ ) (Figure $1(\mathrm{~A})$, Figure $1(\mathrm{~B})$ ). The result showed that the AR extract made a greater impact on cell growth after AR treating the cells.

So as to understand the mechanism of AR more clearly, we chose to analyze the 64 co-DEGs (Figure 1(C)). GO analysis classifies and enriches differential genes from three different dimensions: Biological Process (BP), Cellular Component
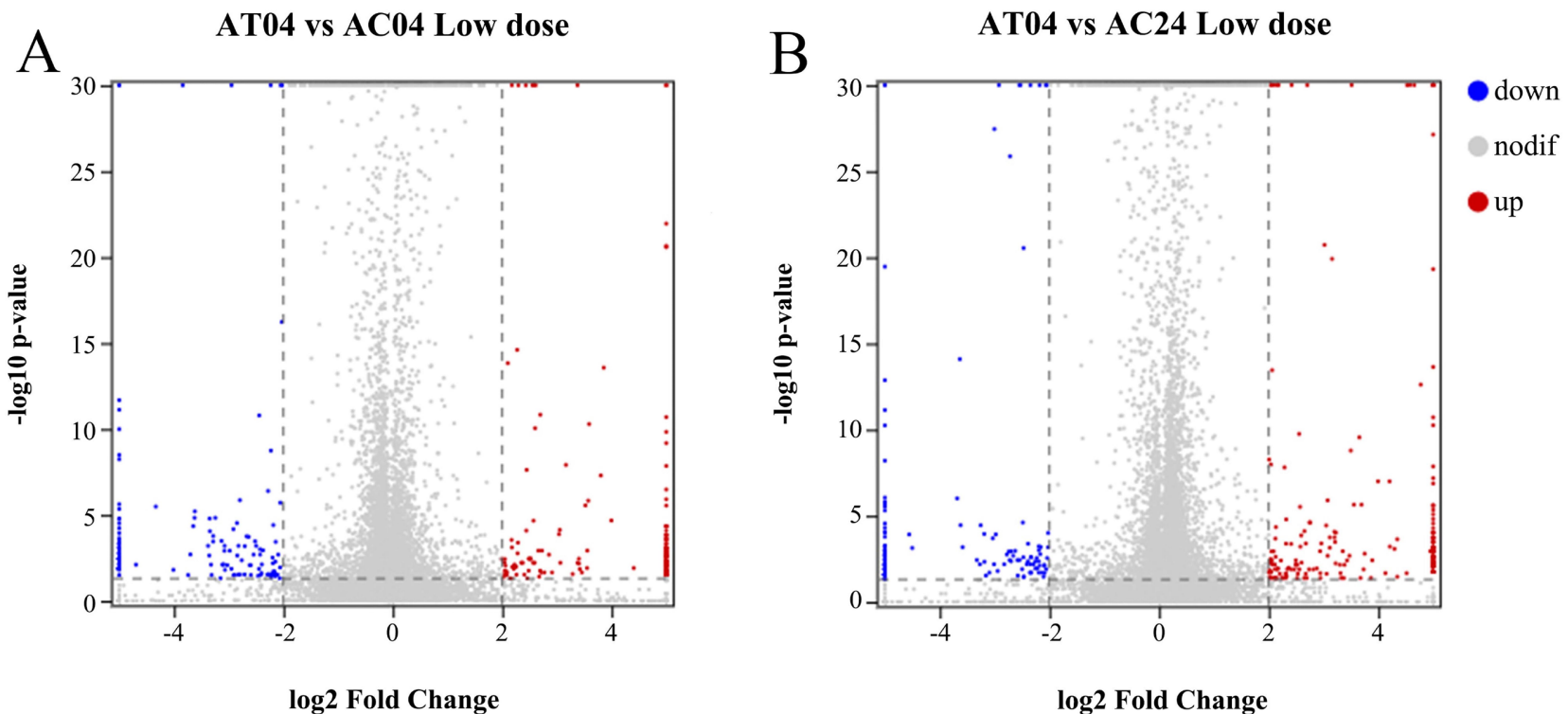
C

$04 \mathrm{~h}$

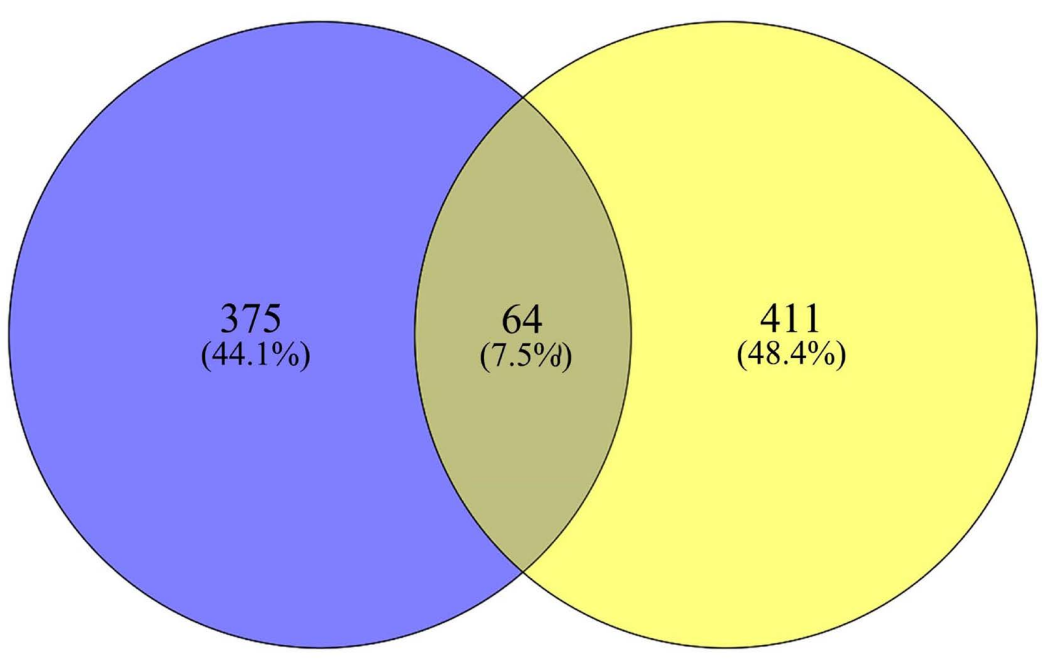

D

GO:0071492 cellular response to UV-A
GO:0045471 response to ethanol
GO:0070141 response to UV-A

GO:0045346 regulation of MHC class II biosynthetic process

GO:0045342 MHC class II biosynthetic process

GO:0002312 B cell activation involved in immune response

GO:0002703 regulation of leukocyte mediated immunity

GO:0009636 response to toxic substance

GO:0046677 response to antibiotic

है

GO:0002313 mature B cell differentiation involved in immune response GO:0002697 regulation of immune effector process

GO:0006464 cellular protein modification process

GO:0036211 protein modification process

GO:0010021 amylopectin biosynthetic process

GO:0030034 microvillar actin bundle assembly GO:0031550 positive regulation of brain-derived neurotrophic factor receptor signaling pathway GO:0072721 cellular response to dithiothreitol GO:0080163 regulation of protein serine/threonine GO:0098694 regulation of synaptic vesicle budding from presynaptic endocytic zone membrane GO:0120186 negative regulation of protein localization to chromatin

\section{Top 20 of GO Enrichment}

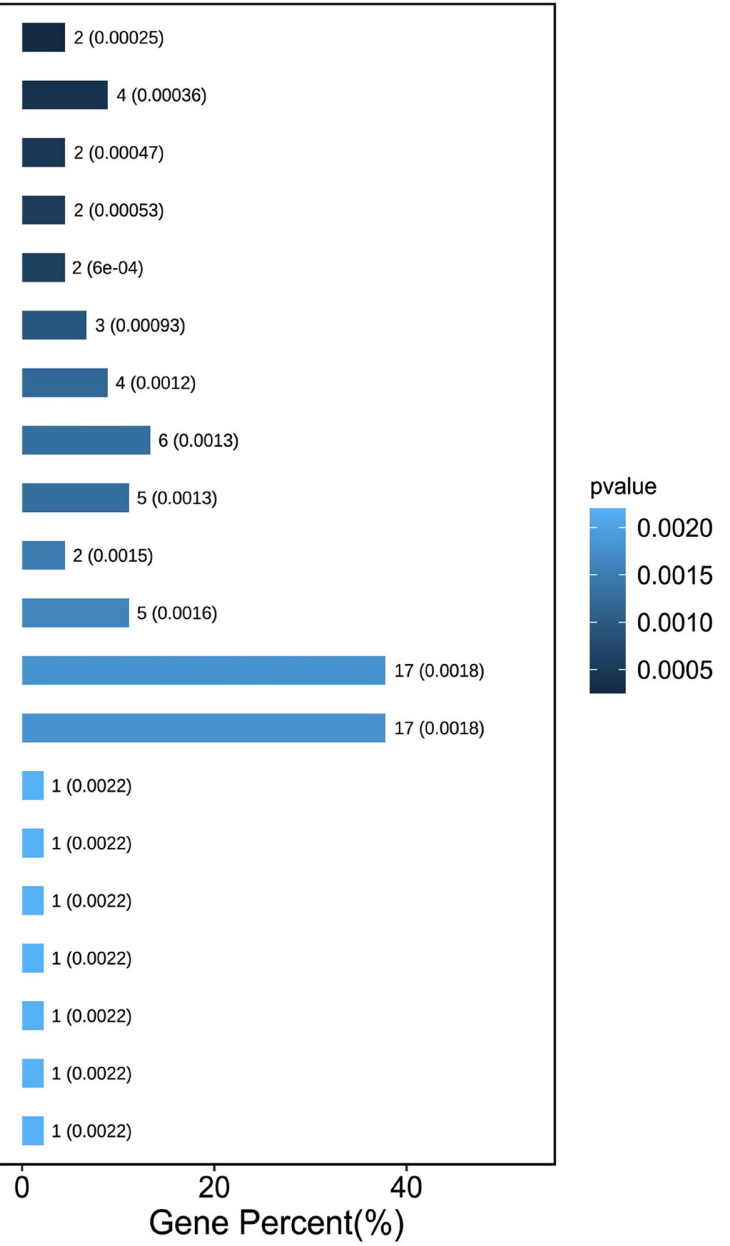


E

Top 20 of GO Enrichment

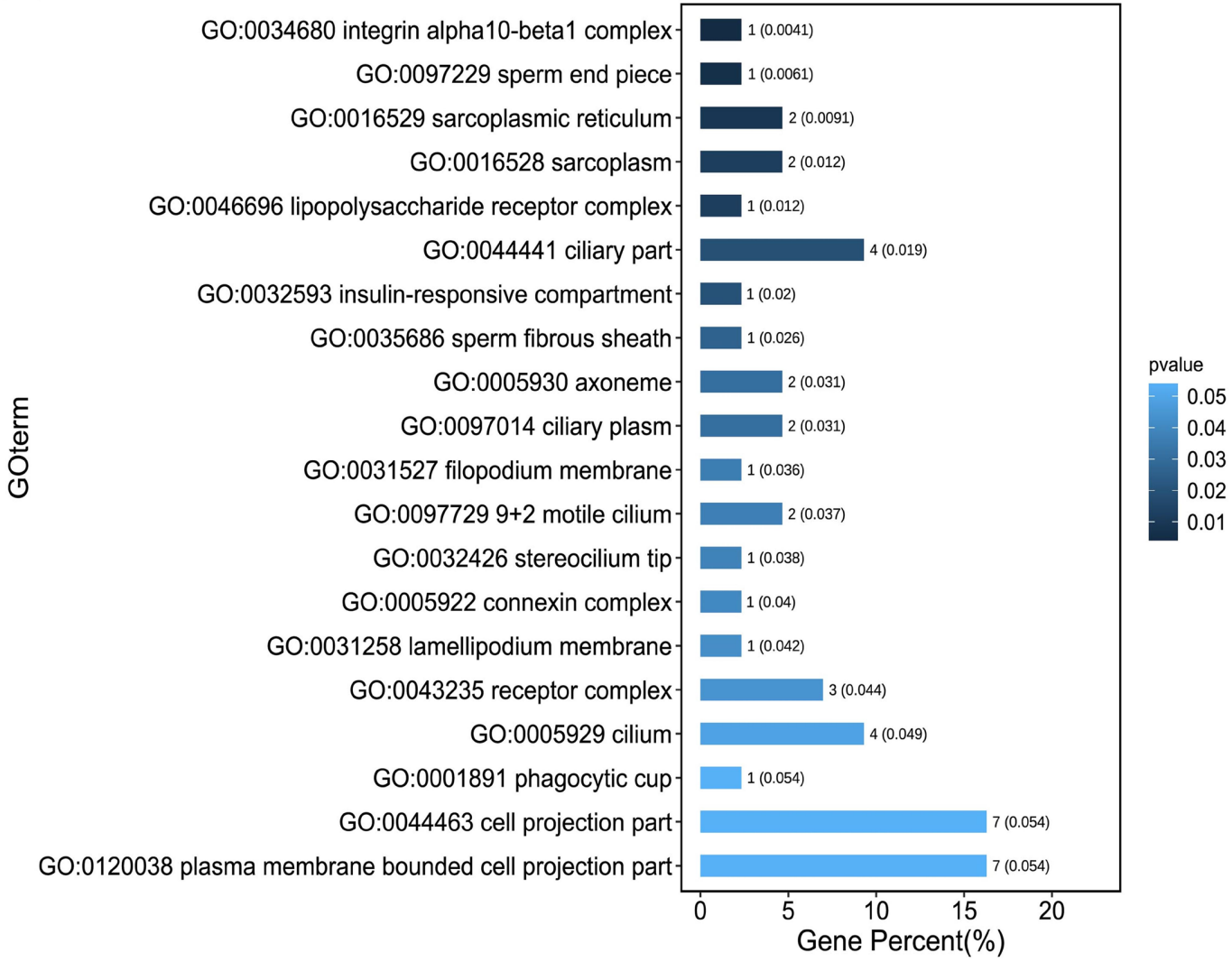

$\mathrm{F}$

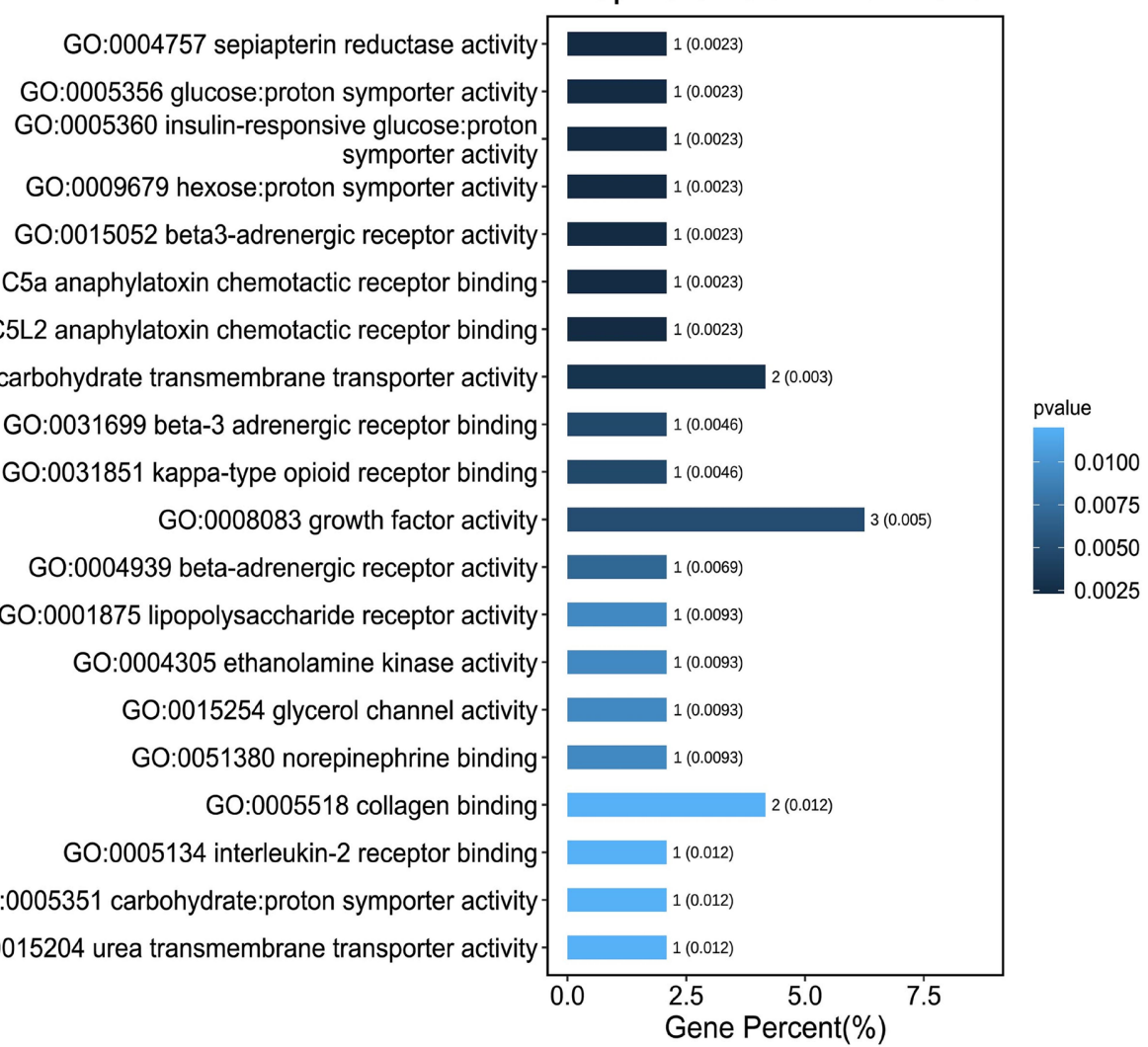




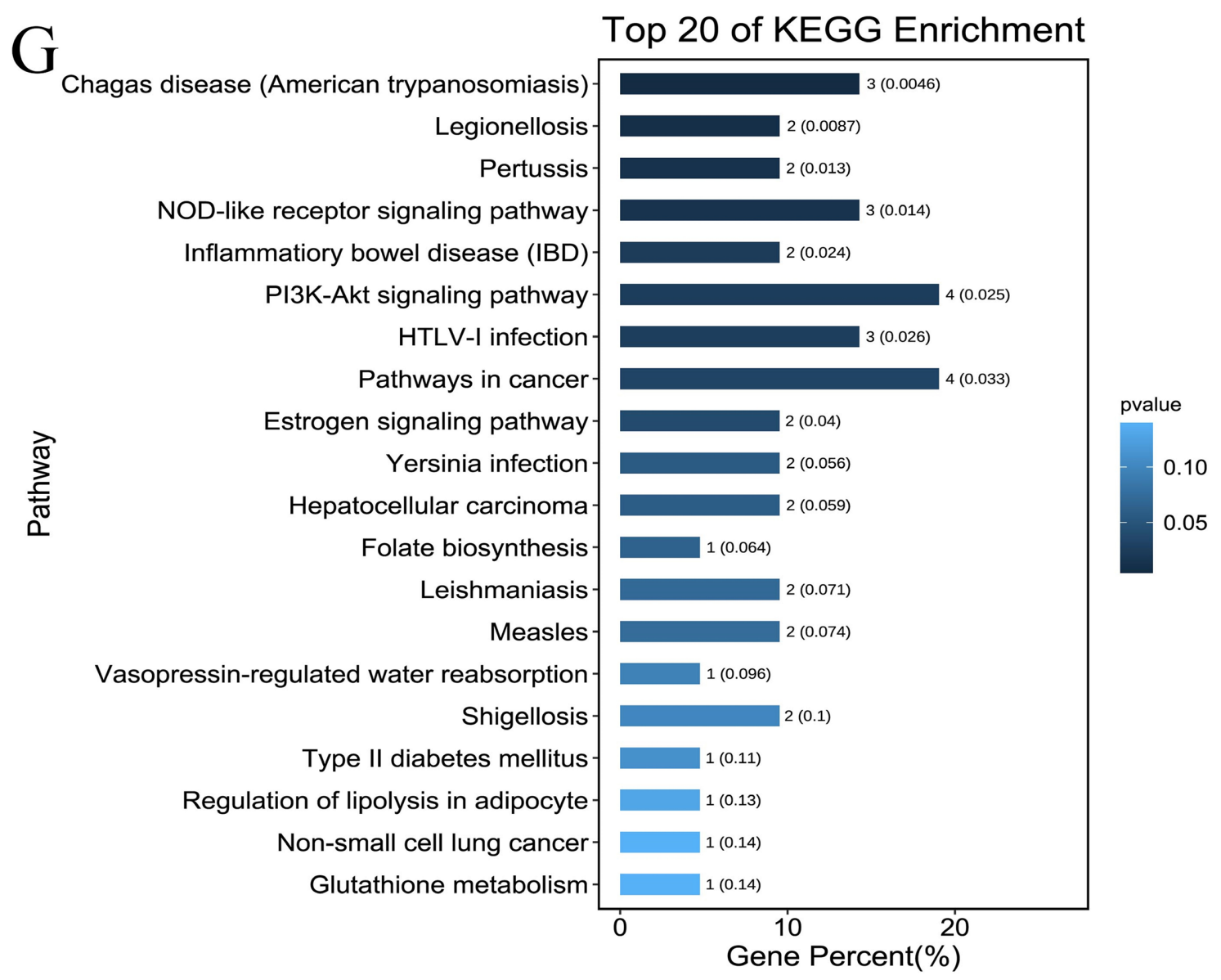

Figure 1. Co-DEGs analysis of low-dose $(175 \mu \mathrm{g} / \mathrm{ml})$ AR extract acting in M-1 cells at different times. (A) Volcano map of DEGs between control group and treatment group at $4 \mathrm{~h}$. (B) Volcano map of DEGs between control group and treatment group at $24 \mathrm{~h}$. (C) Venn map of DEGs between $4 \mathrm{~h}$ and $24 \mathrm{~h}$ under low-dose AR extract on M-1 cell. (D) The top 20 GO terms in the enrichment analysis. (E) The top 20 BP terms in the enrichment analysis. (F) The top $20 \mathrm{CC}$ terms in the enrichment analysis. (G) The top 20 MF terms in the enrichment analysis. (H) The top 20 KEGG pathway in the enrichment analysis.

(CC), and Molecular Function (MF), thereby to reflect the dynamic change process between different groups. It was found that there were more enriched genes in biological regulation, metabolic process and cell development in BP term. The cell part, organelle part, membrane and other enriched genes in CC term were more abundant, and the binding, catalytic activity, molecular function regulation and other enriched genes in MF term were more enriched. The most enriched BP terms included response to UV-A, synthesis and regulation of MHC class II biosynthetic processes (Tlr4, Spi1), response to toxic substances, immune response (Spi1, C3, Rnf26, Il2, Tlr4) and protein modification. The number of enriched genes related to protein modification was the largest among them. Receptor complex (Adrb3, Tlr4, Itga10) and cilium were the most enriched CC terms. The most enriched MF terms were growth factor activity (Cers1, Tgfa, I12), carbohydrate transmembrane transporter activity (Slc2a4, Aqp3) (Figures $1(D)-(F))$. It showed that the AR extract had a greater regulatory effect on cell growth and immune response-related genes after AR acting on cells. 
KEGG pathway enrichment analysis was performed to explore the most significantly enriched DEG pathway (Figure $1(\mathrm{G})$ ). Among the top 20 KEGG pathways, the most enriched were Chagas disease (C3, Il2, Tlr4), NOD-like receptor signaling pathway (Nek7, Antxr1, Tlr4), PI3K-AKT signaling pathway (Il2, Tgfa, Tlr4, Itga10), HTLV-I infection (Spi1, Il2, Rasl2-9), and pathways in cancer. NOD-like receptor signaling pathway played a key role in pathogen recognition and innate immune response; PI3K-AKT signaling pathway was involved in the regulation of many basic cellular processes, indicating that the effects of AR extract on cells changed cellular immune-related genes.

\subsection{Transcriptome Analysis of the Effect of High-Dose AR on M-1 Cells}

The effects of high-dose AR extract on M-1 cells were studied, in which the concentration of AR was increased to 5 times the original $(875 \mu \mathrm{g} / \mathrm{ml})$. As in the previous experiment, the cells were cultured for 4 hours with AR extract, and then the cells were harvested. By comparing the gene expression changes between the control group and treatment group, DEGs were analyzed to identify the effects of high-concentration AR extract on the cells. The experimental results showed that a total of 64 DEGs were detected, of which 35 genes were up-regulated and 29 genes were down-regulated (|fold change $\mid>2$ and $\mathrm{p}<0.05$ ) (Figure 2(A)). The result revealed that the high-concentration AR extract had a significant effect on cell growth after treating the cells.

A

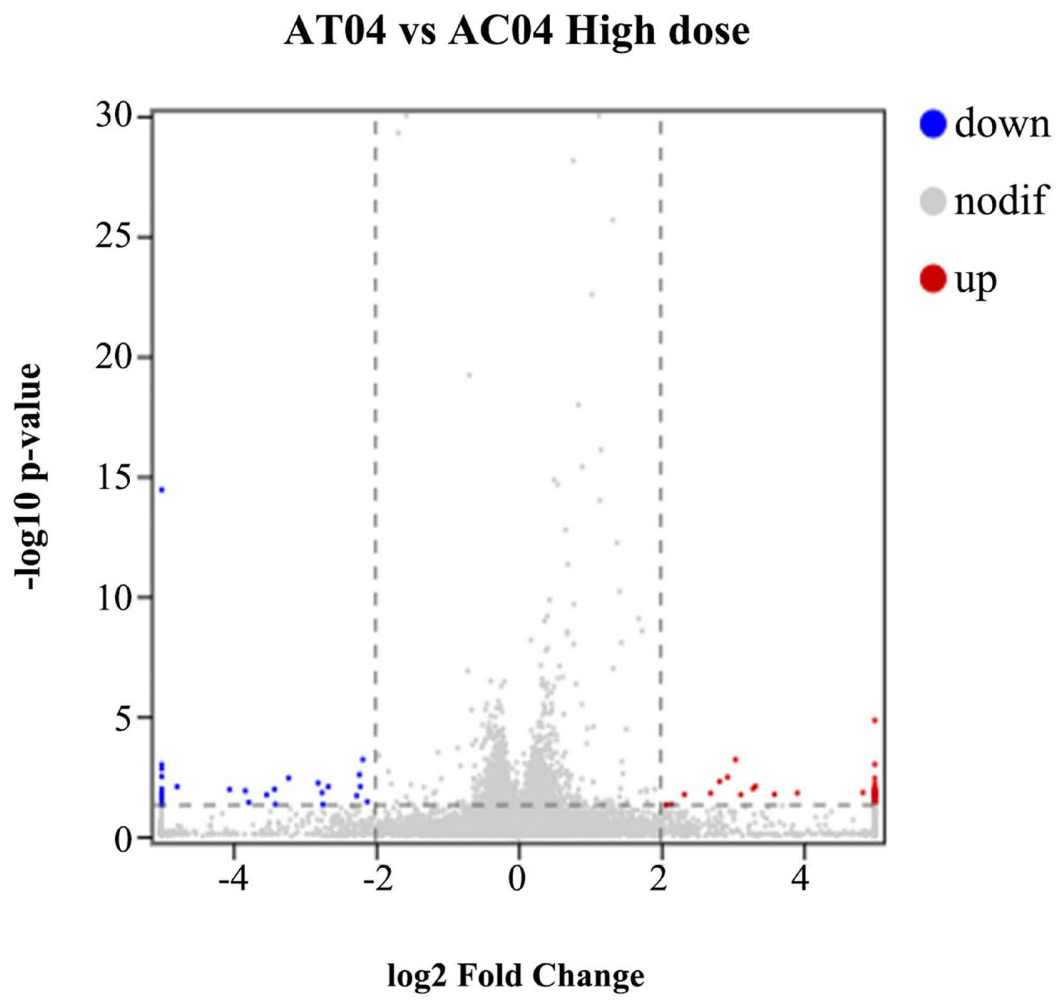




\section{B}

G0:0006335 DNA replication-dependent nucleosome assembly

GO:0034723 DNA replication-dependent nucleosome organization

G0:0002683 negative regulation of immune system process

C

GO:0050687 negative regulation of defense response to virus GO:0048291 isotype switching to $\lg \mathrm{G}$ isotypes megakaryocyte differentiation G0:0048302 regulation of isotype switching to IgG isotypes

GO:0035584 calcium-mediated signaling using intracellular calcium source

GO:0005254 chloride channel activity GO:0005229 intracellular calcium activated chloride channel activity GO:0061778 intracellular chloride channel activity

GO:0005253 anion channel activity

GO:0015108 chloride transmembrane transporter activity GO:0031854 orexigenic neuropeptide QRFP receptor binding

GO:0045509 interleukin-27 receptor activity

GO:0047224 acetylgalactosaminyl-O-glycosyl-glycoprotein beta-1,3-N-acetylglucosaminyltransferase activity GO:0015267 channel activity

GO:0022803 passive transmembrane transporter activity GO:0015103 inorganic anion transmembrane transporter activity GO:0042019 interleukin-23 binding GO:0008009 chemokine activity GO:0022839 ion gated channel activity GO:0022836 gated channel activity GO:0005143 interleukin-12 receptor binding GO:0046982 protein heterodimerization activity GO:0005216 ion channel activity GO:0008237 metallopeptidase activity GO:0042379 chemokine receptor binding

$$
0
$$

Top 20 of GO Enrichment

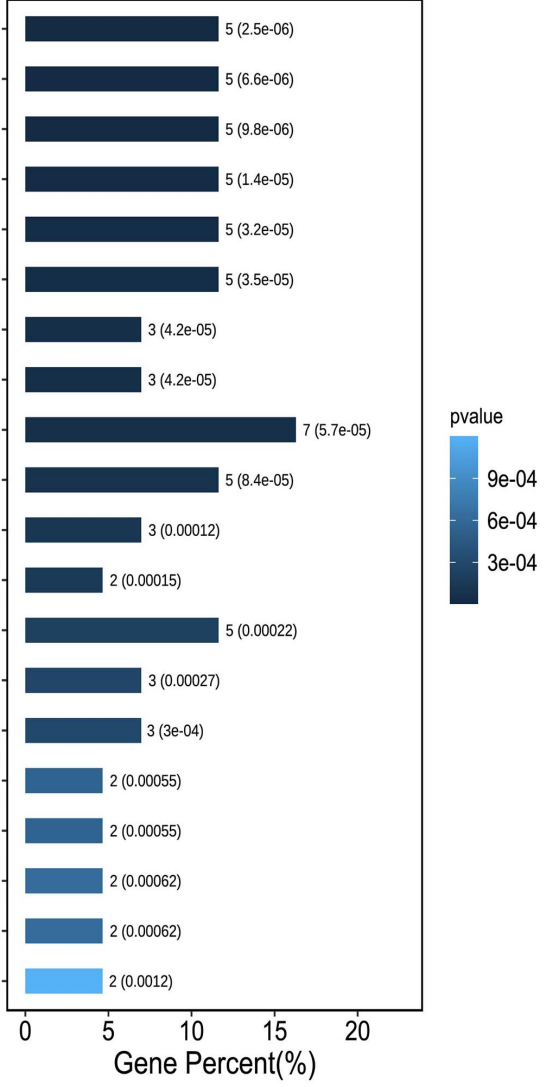

Top 20 of GO Enrichment

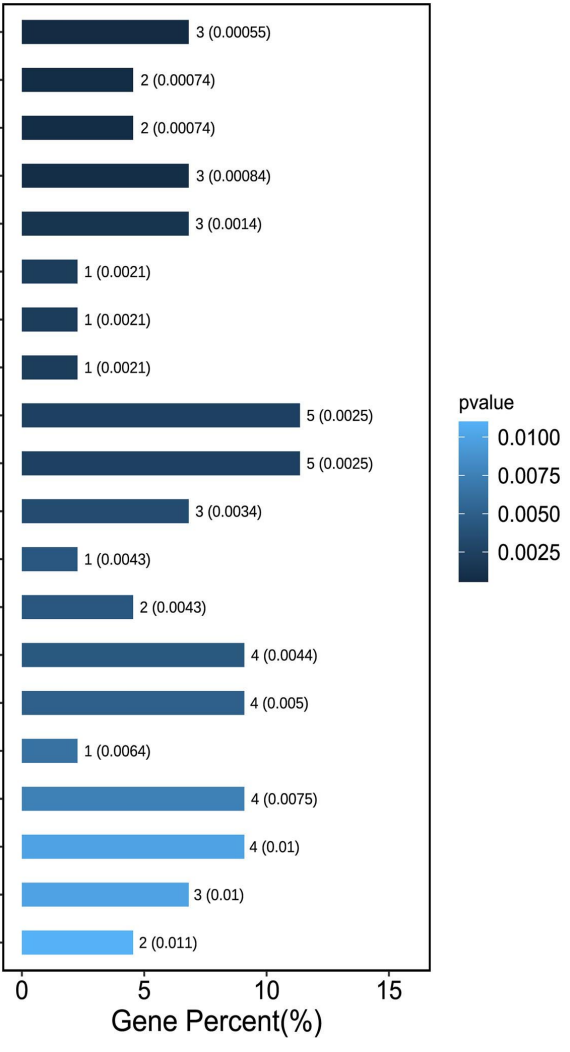


D

Top 20 of GO Enrichment
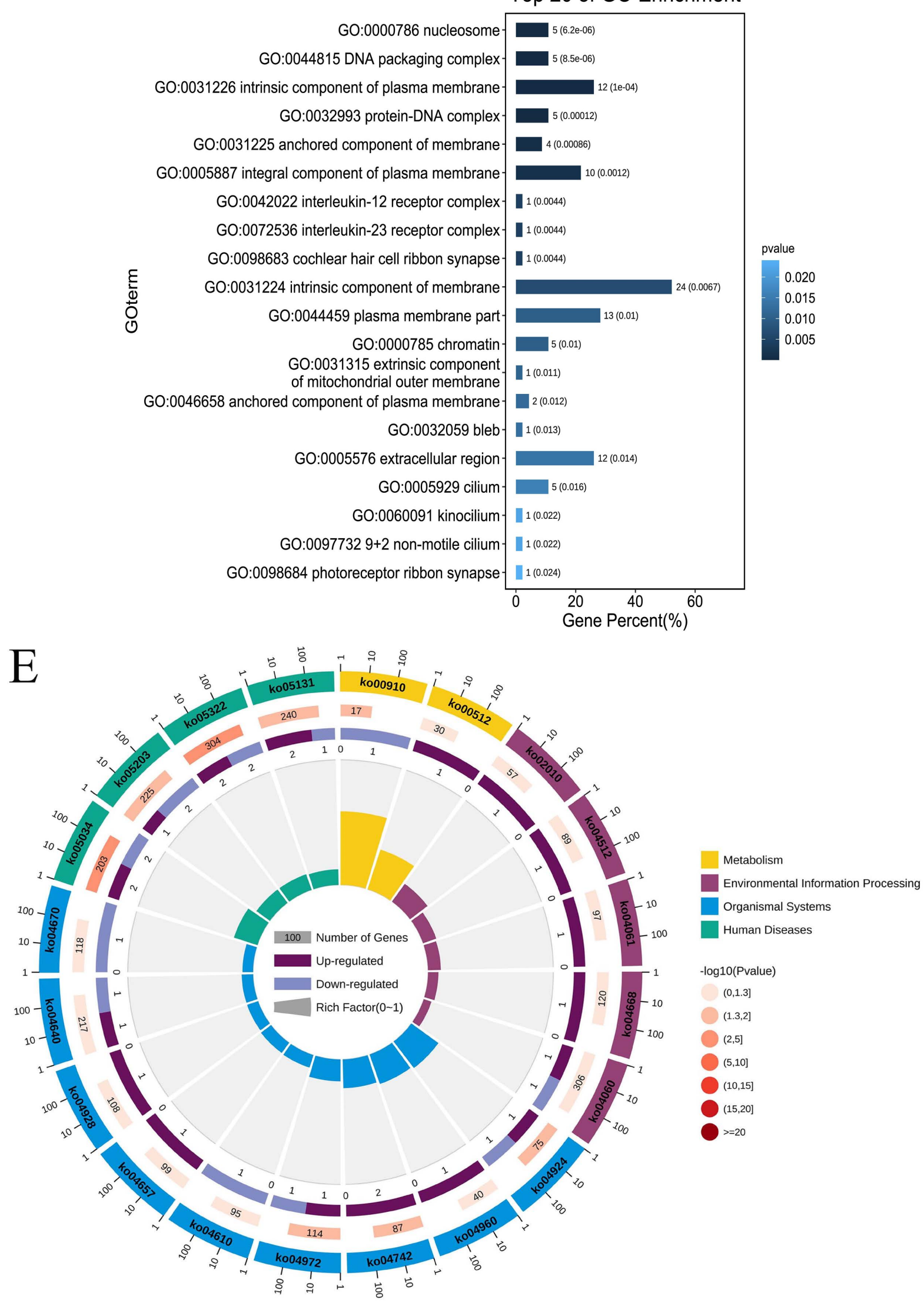


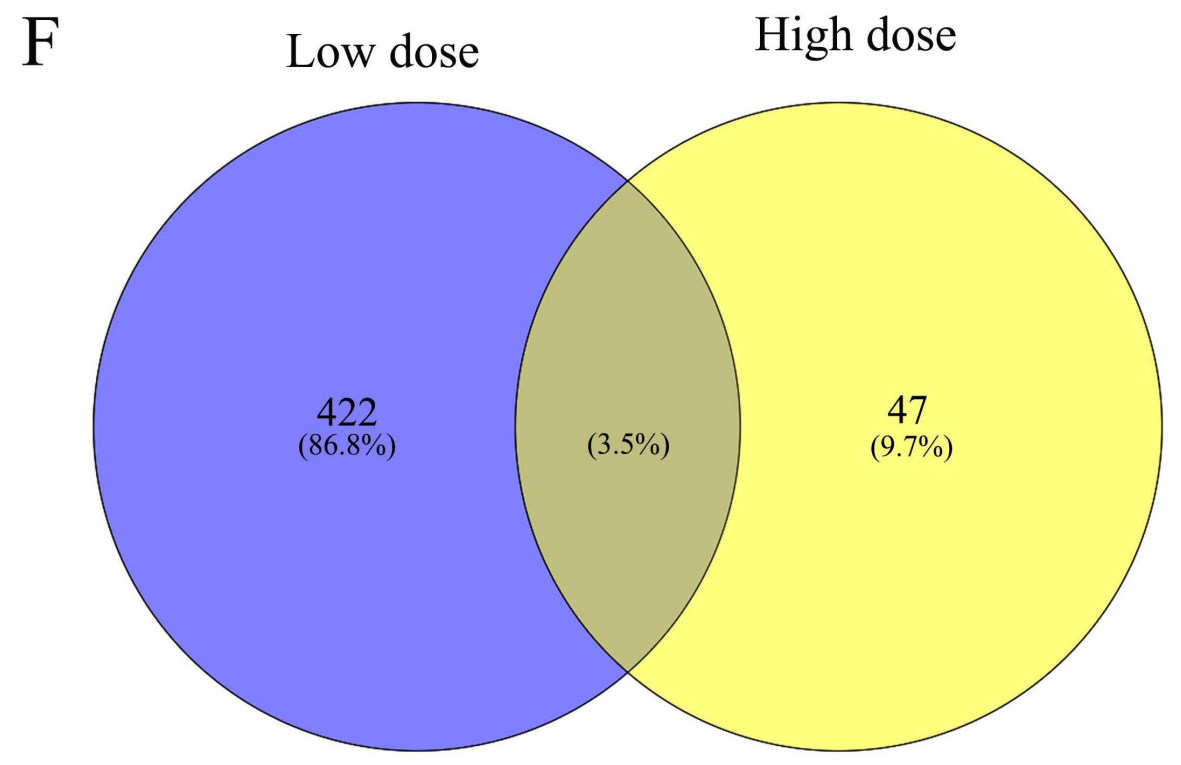

Figure 2. DEGs analysis of high-dose $(875 \mu \mathrm{g} / \mathrm{ml})$ AR extract acting in M-1 cells at $4 \mathrm{~h}$. (A) Volcano map of DEGs between control group and treatment group at $4 \mathrm{~h}$. (B) The top $20 \mathrm{BP}$ terms in the enrichment analysis. (C) The top $20 \mathrm{MF}$ terms in the enrichment analysis. (D) The top $20 \mathrm{CC}$ terms in the enrichment analysis. (E) The top 20 KEGG pathway in the enrichment analysis. (F) Venn map of DEGs between low and high dose of AR extract on M-1 cell at $4 \mathrm{~h}$.

In order to clarify the effective mechanism of the high concentration of AR extract on the growth of cells, the 64 DEGs that were screened were further analyzed. GO analysis showed that BP was mainly concentrated in chromatin, nucleosome assembly (Hist1h1b, Hist1h4c, Hist1h4d, Hist2h3b, Hist2h2bb) and immune system process (Il27ra, Ptprc, Sarm1, Rnf26). CC terms were mainly enriched in the nucleosome (Hist1h1b, Hist1h4c, Hist1h4d, Hist2h3b, Hist2h2bb), intrinsic integral component of plasma membrane (Scnn1g, Gpa33, Il27ra, Ptprc), and anchored component of membrane (Prnd, Mmp17, Lypd5, Car15). The MF terms were enriched in gated channel activity (Clca1, Clcn1, Clca3a1), passive transmembrane transporter activity (Scnn1g, Clcn1, Gjb2, Clca3a1), and protein heterodimerization activity (Hist1h4c, Hist1h4d, Hist2h3b, Hist2h2bb) (Figures 2(B)-(D)). It showed that the high concentration of AR extract mainly acted on basic life processes such as DNA assembly and material transmembrane transportation. Changes in these processes were more likely to change the cell growth state.

KEGG pathway enrichment analysis of differential genes was performed, and the results showed that among the top 20 signaling pathways, the most enriched KEGG pathways were related to diseases such as alcohol abuse, systemic lupus erythematosus, renin secretion, viral carcinogenesis, taste transmission, and Shigellosis (Figure 2(E)). It was also found that the genes enriched in the pathways of alcoholism and Systemic lupus erythematosus were all histones (Table 1). As it was known, histones was involved in the assembly of nucleosomes [10], and histone modifications were the main type of epigenetic modification. Histones were modified by a large number of enzymes and related to a variety of cancers 
Table 1. Top 20 pathways of KEGG enrichment.

\begin{tabular}{|c|c|c|}
\hline ID & Description & Genes Symbol \\
\hline ko05034 & Alcoholism & $\begin{array}{l}\text { Hist1h4c, Hist1h4d, } \\
\text { Hist2h3b, Hist2h2bb }\end{array}$ \\
\hline ko05322 & Systemic lupus erythematosus & $\begin{array}{l}\text { Hist1h4c, Hist1h4d, } \\
\text { Hist2h3b, Hist2h2bb }\end{array}$ \\
\hline ko04924 & Renin secretion & Clca1, Clca3a1 \\
\hline ko05203 & Viral carcinogenesis & $\begin{array}{l}\text { Hist1h4c, Hist1h4d, } \\
\text { Hist2h2bb }\end{array}$ \\
\hline ko04742 & Taste transduction & Scnn1g, Tas1r1 \\
\hline ko05131 & Shigellosis & Myl7, Gjb2, Hist2h3b \\
\hline ko04972 & Pancreatic secretion & Clca1, Clca3a1 \\
\hline ko00910 & Nitrogen metabolism & Car15 \\
\hline ko00512 & Mucin type O-glycan biosynthesis & B3gnt6 \\
\hline ko04960 & Aldosterone-regulated sodium reabsorption & Scnn1g \\
\hline ko04640 & Hematopoietic cell lineage & Gp1bb, Cd55b \\
\hline ko02010 & $\mathrm{ABC}$ transporters & Abca 4 \\
\hline ko04060 & Cytokine-cytokine receptor interaction & Il27ra, Ccl20 \\
\hline ko04512 & ECM-receptor interaction & Gp1bb \\
\hline ko04610 & Complement and coagulation cascades & Cd55b \\
\hline ko04061 & $\begin{array}{l}\text { Viral protein interaction with cytokine } \\
\text { and cytokine receptor }\end{array}$ & $\mathrm{Ccl} 20$ \\
\hline ko04657 & IL-17 signaling pathway & $\mathrm{Ccl} 20$ \\
\hline ko04928 & Parathyroid hormone synthesis, secretion and action & Mmp17 \\
\hline ko04670 & Leukocyte transendothelial migration & Myl7 \\
\hline ko04668 & TNF signaling pathway & $\mathrm{Ccl} 20$ \\
\hline
\end{tabular}

[11] [12], the results showed that AR extract affected the gene expression of histones genes and different concentration of AR had different effect effects, which indicated that AR extract affected the assembly of nucleosomes in multiple manners, which caused the different clinic functions of AR. We performed Venn analysis on the DEGs in the same time period of high dose and low dose, and found a total of 17 co-DEGs (Figure 2(F) and Table 2). 8 co-DEGs (2810468N07Rik, Il27ra, Dnah5, AC123954.1, Pcdhgc4, Gp1bb, Clcn1, Ccl20) were found to have opposite expression levels in the two groups. The genes expression of high dose AR among these co-DEGs were positively correlated with the occurrence of diseases, which demonstrated that the KEGG pathways altered by high-concentration AR extract were related to the occurrence of diseases, and had side effects on cells growth. 
Table 2. co-DEGs of low and high dose AR in M-1 (AT04 vs AC04).

\begin{tabular}{|c|c|c|c|}
\hline Genes Symbol & Gene ID & Low dose & High dose \\
\hline Prnd & ENSMUSG00000027338 & 12.49022414 & 2.935376615 \\
\hline U90926 & ENSMUSG00000029409 & 12.01272928 & 12.55300364 \\
\hline 2810468N07Rik & ENSMUSG00000091475 & 10.95329434 & -2.664937743 \\
\hline Il27ra & ENSMUSG00000005465 & 9.865114619 & -9.376436547 \\
\hline Mmp17 & ENSMUSG00000029436 & 8.970767394 & 8.159787261 \\
\hline Dnah5 & ENSMUSG00000022262 & 7.333065878 & -2.225834753 \\
\hline Gpa33 & ENSMUSG00000000544 & 2.512943944 & 2.065910967 \\
\hline AC123954.1 & ENSMUSG00000113255 & -2.026644561 & 2.080883822 \\
\hline Pcdhgc4 & ENSMUSG00000023036 & -2.094222386 & 2.330155403 \\
\hline $\operatorname{Rnf} 26$ & ENSMUSG00000053128 & -5.923689501 & -5.339661043 \\
\hline Gp1bb & ENSMUSG00000050761 & -9.056897033 & 5.74293525 \\
\hline Clcn 1 & ENSMUSG00000029862 & -9.577372177 & 9.583446374 \\
\hline A430105J06Rik & ENSMUSG00000097784 & -11.07984475 & -11.01433669 \\
\hline Cd55b & ENSMUSG00000026401 & -11.67142607 & -11.22068868 \\
\hline Prrx2 & ENSMUSG00000039476 & -11.68359558 & -3.52890236 \\
\hline $\mathrm{Ccl} 20$ & ENSMUSG00000026166 & -11.86405843 & 3.292019206 \\
\hline A530084C06Rik & ENSMUSG00000090863 & -12.11355661 & -10.13644007 \\
\hline
\end{tabular}

\section{Discussion}

AR is a traditional Chinese medicine for invigorating Qi that is widely used in clinical Chinese medicine, and its clinical efficacy has been universally recognized by modern medicine [13]. Qi is a relatively abstract concept in traditional Chinese medicine. The theory of traditional Chinese medicine believes that Qi is the driving force of human life activities and one of the basic substances that maintain the normal life activities of the human body from different aspects. Studies have shown that AR extract has complex components, including Astragaloside, Astragalus flavonoids, APS and amino acids, which play a role in immune regulation, multi-organ protection, hypoglycemia, anti-virus, and antitumor [13] [14]. As so far, the cellular biological mechanism of AR curative effect is not clear.

In order to understand the AR effects on cells through modern technology, we used AR extract to treat cells for different times and concentrations. With the help of transcriptomics technology, we analyzed the changes in cell gene transcription levels and reveal the significance of AR in cell life activities. The results showed that treatment of cells with a low concentration of AR extract can screen out prolific DEGs, and the co-DEGs of different treatment times were mainly enriched in cell growth, metabolic processes, immune response and other related pathways, which were consistent with the multiple clinical function of AR in Chinese medicine [13]. After a high dose of AR acts on cells, its signal pathways 
are mainly concentrated on disease-related pathways such as systemic lupus erythematosus, viral carcinogenesis, and Shigellosis. The results well explained that the high dose of AR extract may cause some side-effect in healthy people.

The growth and development of cells is usually a complex process involving various regulatory molecules. Cell growth depends on the comprehensive effects of biochemical and molecular processes that synthesize its various components inside the cell. In our previous experimental results, it was found that low dose of AR increased the content of ATP and GSH in cells [15]. ATP is the carrier of energy released by cells during respiration and directly provides energy for various life activities. Mitochondrial homeostasis is important for maintaining mitochondrial function and cell stability [16]. Low concentration of AR acted on the differential genes screened by cells, and multiple genes become potential target genes, such as ceramide synthase 1 (Cers1), aquaporin 3 (Aqp3), Interleukin-2 (Il2) and so on (Result 3.1). Cers1 is a transduction stress signal that regulates lipid metabolism from the endoplasmic reticulum to the outer mitochondrial membrane [17]. The transmembrane transport of $\mathrm{H}_{2} \mathrm{O}_{2}$ requires the participation of Aqp. $\mathrm{H}_{2} \mathrm{O}_{2}$ crosses the cytoplasmic membrane from outside the cell into the cytoplasm with the assistance of Aqp3 [18]. Aqp3 can also inhibit cell apoptosis by reducing the genes expression related Wnt/GSK- $3 \beta / \beta$-catenin pathway. Toll-like receptor $4(\mathrm{Tl} 4)$ participates in a variety of autoimmune diseases by acting as a bridge between innate immunity and acquired immunity. The activation of Tlr4 induces mitochondrial dynamic imbalance and promotes the occurrence of diseases [19]. NIMA-related kinase 7 (Nek7) is a multifunctional kinase that affects centrosome replication, mitochondrial regulation, intracellular protein transport, DNA repair and mitotic spindle assembly [20], and participates in NF- $\kappa$ B signaling. (I12) is a pro-inflammatory cytokine that can regulate cell growth, proliferation, and apoptosis. After Il2 stimulation, the quality of mitochondria changes, or the production of ROS in cells increases [21] [22]. In addition, there are some differential gene functions related to mitochondria, such as the perredoxin (Prx) family that controls the level of $\mathrm{H}_{2} \mathrm{O}_{2}$ in the cell and regulates signal transduction [23]. Glutathione S-transferase (GST) family members 7 (Gstm7) exerts the detoxification effect of electrophilic compounds by binding to reduced glutathione [24]. The transcription of E3 ligase proteins Fbxo15 and Ring finger protein 26 (Rnf26) is involved in the immune regulation function of cells [25] [26]. These genes are directly and indirectly related to the function of mitochondria. And it can be seen from Figure 1, E that in the BP of co-DEGs, protein modification related genes had the most enrichment. It showed that low concentrations of AR may play a role in changing the function of mitochondria by modifying the protein after expression of mitochondrial-related genes, thereby improving cellular immune function and cell growth.

The decrease of mitochondrial membrane potential can cause a series of biochemical changes in the mitochondrial membrane, which is an early event in the 
process of cell apoptosis [27]. High concentration of AR reduces the production of ATP and lowers the membrane potential of mitochondria [15]. Excessive mitochondrial fission can cause mitochondrial ROS overload and inhibit the production of mitochondrial ATP [28]. As it showed in Table 1, the high concentration of AR extract produced drug toxicity for cell growth and caused cellular immune response. Among the co-DEGs shared with the low-concentration AR extract, the expression levels of multiple genes showed opposite results (Table 2 ). The expression patterns of these genes under the action of high concentrations of AR were mostly related to the occurrence of diseases. Studies have found that human neutrophils treated with Interleukin-27 (I127) swelled the cells, ruptured mitochondria and condensed the nucleus [29]. Chemokine (C-C motif) ligand $20(\mathrm{Ccl} 20)$ is expressed in various human tissues and immune cells, and involved in local immune cell recruitment [30]. Ccl20 is significantly up-regulated in a variety of cancer tissues. Ccl20 produced by liver cancer cells recruits Ccr6+CD5+B cells and induces blood vessels generated to promote tumor growth [31]. In our experiments, it was also found that Ccl20 expression was highly expressed under the action of high concentrations of AR. The mutation of Clcn1 is related to myotonia [32]. GPIb-IX-V complex is expressed on megakaryocytes and platelets, which acts as a receptor for von Willebrand factor and mediates platelet-subendothelial interactions, and is a major participant in hemostasis. The glycoprotein Ib (GpIb)-IX-V complex contains four different transmembrane subunits: GpIba, GpIb $\beta$, GpIX and Gpv. Mutations in GpIb $\beta$ caused GpIbIX-V defects [33]. Some experiments have found that high concentrations of AR can shorten the lifespan of bees and also have an inhibitory effect on the growth of cancer cells [34] [35]. It was obvious that high concentrations of drugs had adverse effects on cells and may promote the occurrence of diseases. In addition, the high dose of AR extract also changes the expression of multiple histones, which can cause some genes to change their expression patterns at the DNA level. This also shows that the high concentration of AR extract may have drug toxicity on cells, resulting in changes in gene expression patterns related to diseases.

Based on the RNA-Seq analysis, we gained further understanding of the molecular mechanism of $\mathrm{AR}$, and also learned the preliminary functional mechanism for the side effects of the high drug concentration AR. Several genes were identified as potential targets of $\mathrm{AR}$, which were expected to become treatment strategies for diseases caused by abnormal expression of them. Our future research will focus on animal model to verify these hypotheses.

\section{Conclusion}

Using RNA-Seq, the DEGs related to the effects of the AR extract on the cells were identified. The GO and KEGG function enrichment analysis of DEGs showed that a low concentration of AR extract was beneficial to cell growth and enhance the expression of genes related to cellular immune response. Many genes 
are related to mitochondrial function, and high concentrations of AR may cause abnormal expression of multiple disease-related genes in cells. Here, for the first time, we use AR extract to directly act on healthy cells, and explain the role of AR from the perspective of mitochondria. These results have a guiding role in accurately understanding the pharmacological effects of AR and its molecular mechanism, which is helpful for application of AR extract in disease prevention and treatment.

\section{Acknowledgements}

We thank the ENN Group for providing the funding for this research. Bioinformatic analysis was performed using the OmicShare tools, a free online platform for data analysis. We are also grateful to the editor and anonymous reviewers for their constructive criticisms and suggestions, which significantly improved this manuscript.

\section{Conflicts of Interest}

The authors declare no conflicts of interest regarding the publication of this paper.

\section{References}

[1] Adhikari, B., Marasini, B.P., Rayamajhee, B. and Bhattarai, B.R. (2021) Potential Roles of Medicinal Plants for the Treatment of Viral Diseases Focusing on COVID-19: A Review. Phytotherapy Research, 35, 1298-1312. https://doi.org/10.1002/ptr.6893

[2] Tan, Y.Q., Chen, H.W. and Li, J. (2020) Astragaloside IV: An Effective Drug for the Treatment of Cardiovascular Diseases. Drug Design, Development and Therapy, 14, 3731-3746. https://doi.org/10.2147/DDDT.S272355

[3] Hostetler, G.L., Ralston, R.A. and Schwartz, S.J. (2017) Flavones: Food Sources, Bioavailability, Metabolism, and Bioactivity. Advances in Nutrition, 8, 423-435. https://doi.org/10.3945/an.116.012948

[4] Wu, C.Y., Ke, Y., Zeng, Y.F. and Zhang, Y.W. (2017) Anticancer Activity of Astragalus polysaccharide in Human Non-Small Cell Lung Cancer Cells. Cancer Cell International, 17, 115. https://doi.org/10.1186/s12935-017-0487-6

[5] Zheng, Y., Ren, W., Zhang, L. and Zhang, Y. (2020) A Review of the Pharmacological Action of Astragalus polysaccharide. Frontiers in Pharmacology, 11, Article No. 349. https://doi.org/10.3389/fphar.2020.00349

[6] Dong, N., Li, X., Xue, C. and Zhang, L. (2020) Astragalus polysaccharides Alleviates LPS-Induced Inflammation via the NF- $\mathrm{kB} / \mathrm{MAPK}$ Signaling Pathway. Journal of Cellular Physiology, 235, 5525-5540. https://doi.org/10.1002/jcp.29452

[7] Zhang, Y., Tao, C., Xuan, C. and Jiang, J. (2020) Transcriptomic Analysis Reveals the Protection of Astragaloside IV against Diabetic Nephropathy by Modulating Inflammation. Oxidative Medicine and Cellular Longevity, 2020, Article ID: 9542165. https://doi.org/10.1155/2020/9542165

[8] Mhiri, W., Ceylan, M., Turgut-Kara, N. and Nalbantoglu, B. (2020) Transcriptomic Analysis Reveals Responses to Cycloastragenol in Arabidopsis thaliana. PLoS ONE, 15, e0242986. https://doi.org/10.1371/journal.pone.0242986 
[9] Lu, T., Bian, Y., Zhu, Y. and Guo, M. (2020) HUANGKUISIWUFANG Inhibits Pyruvate Dehydrogenase to Improve Glomerular Injury in Anti-Thyl Nephritis Model. Journal of Ethnopharmacology, 253, Article ID: 112682. https://doi.org/10.1016/j.jep.2020.112682

[10] Fyodorov, D.V., Zhou, B.R., Skoultchi, A.I. and Bai, Y. (2018) Emerging Roles of Linker Histones in Regulating Chromatin Structure and Function. Nature Reviews Molecular Cell Biology, 19, 192-206. https://doi.org/10.1038/nrm.2017.94

[11] Karsli-Ceppioglu, S., Dagdemir, A., Judes, G. and Ngollo, M. (2014) Epigenetic Mechanisms of Breast Cancer: An Update of the Current Knowledge. Epigenomics, 6, 651-664. https://doi.org/10.2217/epi.14.59

[12] Xie, W., Zhang, J., Zhong, P. and Qin, S. (2019) Expression and Potential Prognostic Value of Histone Family Gene Signature in Breast Cancer. Experimental and Therapeutic Medicine, 18, 4893-4903. https://doi.org/10.3892/etm.2019.8131

[13] Zhang, C.H., Yang, X., Wei, J.R. and Chen, N.M. (2021) Ethnopharmacology, Phytochemistry, Pharmacology, Toxicology and Clinical Applications of Radix Astragali. Chinese Journal of Integrative Medicine, 27, 229-240. https://doi.org/10.1007/s11655-019-3032-8

[14] Liu, P., Zhao, H. and Luo, Y. (2017) Anti-Aging Implications of Astragalus Membranaceus (Huangqi): A Well-Known Chinese Tonic. Aging and Disease, 8, 868-886. https://doi.org/10.14336/AD.2017.0816

[15] Chen, Y., Feng, Q., Li, M. and Cai, Z. (2020) The Effect of TCM Herbs on Mitochondrial Functions: The Linkage between Qi and Mitochondria. Chinese Medicine, 11, 127-141. https://doi.org/10.4236/cm.2020.114008

[16] Youle, R.J. and van der Bliek, A.M. (2012) Mitochondrial Fission, Fusion, and Stress. Science, 337, 1062-1065. https://doi.org/10.1126/science.1219855

[17] Oleinik, N., Kim, J., Roth, B.M. and Selvam, S.P. (2019) Mitochondrial Protein Import Is Regulated by p17/PERMIT to Mediate Lipid Metabolism and Cellular Stress. Science Advances, 5, eaax1978. https://doi.org/10.1126/sciadv.aax1978

[18] Martinotti, S., Laforenza, U., Patrone, M. and Moccia, F. (2019) Honey-Mediated Wound Healing: $\mathrm{H}_{2} \mathrm{O}_{2}$ Entry through AQP3 Determines Extracellular $\mathrm{Ca}^{2+}$ Influx. International Journal of Molecular Sciences, 20, Article No. 764. https://doi.org/10.3390/ijms20030764

[19] Wu, B., Li, J., Ni, H. and Zhuang, X. (2018) TLR4 Activation Promotes the Progression of Experimental Autoimmune Myocarditis to Dilated Cardiomyopathy by Inducing Mitochondrial Dynamic Imbalance. Oxidative Medicine and Cellular Longevity, 2018, Article ID: 3181278. https://doi.org/10.1155/2018/3181278

[20] Liu, G., Chen, X., Wang, Q. and Yuan, L. (2020) NEK7: A Potential Therapy Target for NLRP3-Related Diseases. BioScience Trends, 14, 74-82.

https://doi.org/10.5582/bst.2020.01029

[21] Miranda, D., Jara, C., Mejias, S. and Ahumada, V. (2018) Deficient Mitochondrial Biogenesis in IL-2 Activated NK Cells Correlates with Impaired PGC1-Alpha Upregulation in Elderly Humans. Experimental Gerontology, 110, 73-78. https://doi.org/10.1016/j.exger.2018.05.014

[22] Qian, J., Fang, D., Lu, H. and Cao, Y. (2018) Tanshinone IIA Promotes IL2-Mediated SW480 Colorectal Cancer Cell Apoptosis by Triggering INF2-Related Mitochondrial Fission and Activating the Mst1-Hippo Pathway. Biomedicine \& Pharmacotherapy, 108, 1658-1669. https://doi.org/10.1016/j.biopha.2018.09.170

[23] Collins, J.A., Wood, S.T., Bolduc, J.A. and Nurmalasari, N.P.D. (2019) Differential Peroxiredoxin Hyperoxidation Regulates MAP Kinase Signaling in Human Articu- 
lar Chondrocytes. Free Radical Biology and Medicine, 134, 139-152. https://doi.org/10.1016/j.freeradbiomed.2019.01.005

[24] Bogdani, M., Henschel, A.M., Kansra, S. and Fuller, J.M. (2013) Biobreeding Rat Islets Exhibit Reduced Antioxidative Defense and $N$-Acetyl Cysteine Treatment Delays Type 1 Diabetes. Journal of Endocrinology, 216, 111-123. https://doi.org/10.1530/JOE-12-0385

[25] Fenech, E.J., Lari, F., Charles, P.D. and Fischer, R. (2020) Interaction Mapping of Endoplasmic Reticulum Ubiquitin Ligases Identifies Modulators of Innate Immune Signalling. Elife, 9, e57306. https://doi.org/10.7554/eLife.57306

[26] Zhang, Z.X., Wang, Y., Shan, Y.T. and Zhou, R. (2020) Oroxylin A Alleviates Immunoparalysis of CLP Mice by Degrading CHOP through Interacting with FBXO15. Scientific Reports, 10, Article No. 19272.

https://doi.org/10.1038/s41598-020-76285-x

[27] Pan, P., Wang, X. and Liu, D. (2018) The Potential Mechanism of Mitochondrial Dysfunction in Septic Cardiomyopathy. Journal of International Medical Research, 46, 2157-2169. https://doi.org/10.1177/0300060518765896

[28] Shi, C., Cai, Y., Li, Y.H., Li, Y., et al. (2018) Yap Promotes Hepatocellular Carcinoma Metastasis and Mobilization via Governing Cofilin/F-Actin/Lamellipodium Axis by Regulation of JNK/Bnip3/SERCA/CaMKII Pathways. Redox Biology, 14, 59-71. https://doi.org/10.1016/j.redox.2017.08.013

[29] Li, J.P., Wu, H., Xing, W. and Yang, S.G. (2010) Interleukin-27 as a Negative Regulator of Human Neutrophil Function. Scandinavian Journal of Immunology, 72, 284-292. https://doi.org/10.1111/j.1365-3083.2010.02422.x

[30] Tournadre, A. and Miossec, P. (2012) Interleukin-17 in Inflammatory Myopathies. Current Rheumatology Reports, 14, 252-256. https://doi.org/10.1007/s11926-012-0242-x

[31] Kadomoto, S., Izumi, K. and Mizokami, A. (2020) The CCL20-CCR6 Axis in Cancer Progression. International Journal of Molecular Sciences, 21, Article No. 5186. https://doi.org/10.3390/ijms21155186

[32] Gaitan-Penas, H., Armand-Ugon, M., Macaya, A. and Estevez, R. (2018) CLCN1 Myotonia congenita Mutation with a Variable Pattern of Inheritance Suggests a Novel Mechanism of Dominant Myotonia. Muscle \& Nerve, 58, 157-160. https://doi.org/10.1002/mus.26098

[33] Rao, A.K. and Songdej, N. (2017) Parsing the Repertoire of GPIb-IX-V Disorders. Blood, 129, 403-404. https://doi.org/10.1182/blood-2016-12-753186

[34] Hong, T., Li, L.X., Han, X.P. and Shi, J.L. (2020) Effect of Astragalus membranaceus Oral Solution on Lifespan and Learning and Memory Ability of Honey Bees. BioMed Research International, 2020, Article ID: 5745048. https://doi.org/10.1155/2020/5745048

[35] Lai, X.Y., Xia, W.B., Wei, J. and Ding, X.H. (2017) Therapeutic Effect of Astragalus Polysaccharides on Hepatocellular Carcinoma H22-Bearing Mice. Dose-Response, 15, Article ID: 1559325816685182 . https://doi.org/10.1177/1559325816685182 